\title{
ON NILPOTENCY IN NONASSOCIATIVE ALGEBRAS
}

\section{J. A. BÉRÉ, M. F. OUEDRAOGO and M. OUATTARA}

Laboratoire T. N. AGATA/UFR-SEA

Département de Mathématiques

Université de Ouagadougou

Adresse 03 B. P. 7021 Ouagadougou

Burkina Faso 03

e-mail: bere_jean0@yahoo.fr

omfrancoise@yahoo.fr

ouatt_ken@yahoo.fr

\begin{abstract}
If a non associative algebra $A$ is right nilpotent (resp. left nilpotent) of degree $n$, then it is strongly nilpotent of degree less or equal to $4 n^{2}-2 n+1$.
\end{abstract}

\section{Introduction}

It is well known that an associative algebra $A$ is nilpotent if for some positive integer $n$, every product of $n$ or more elements of $A$ vanishes. For a nonassociative algebra $A$, we must indicate in which order the products are made. It follows that the products of $n$ elements of $A$ could be zero for a choice of arrangements of parentheses and different from zero for 2010 Mathematics Subject Classification: 17D10.

Keywords and phrases: (non)associative algebra, left nilpotency, right nilpotency, nilpotency, strong nilpotency, index of nilpotency.

Received October 8, 2016

(C) 2016 Scientific Advances Publishers 
another choice. This fact led to the definition of various concepts of nilpotency, namely, left nilpotency, right nilpotency, nilpotency and strong nilpotency, ....

In [5, Proposition 1, p. 82], the authors give the following:

Proposition 1.1. Let $A$ be a commutative (or anticommutative) algebra. Then $A^{\left\{2^{n}\right\}} \subseteq A^{n}$ for any $n \geq 1$. In the other words, if the algebra $A$ is right nilpotent of index $n$, then it is nilpotent of index not greater than $2^{n}$.

We give a generalization of this result to all nonassociative algebras and by the way we show that the boundary of the index is polynomial, namely, the boundary is less or equal to $4 n^{2}-2 n+1$, which is better than $2^{n}$. The main theorem is already done for Malcev and Leibniz algebras (see [1, 2]).

There is Leibniz algebras that are left nilpotent and not right nilpotent, see, for example, [4, Example 3.3].

Using the notion of $A s s o_{k}$-right nil (resp. Asso ${ }_{k}$-left nil), we prove that if an ideal $B$ of a nonassociative algebra $A$ is right nilpotent (resp. left nilpotent) of degree $n$, then $B$ is strongly nilpotent of degree less or equal to $4 n^{2}-2 n+1$.

In Section 2, we give some definitions that we will use along the paper, then in the following Section 3, we prove some results on right products of length $n$.

The Section 4 is devoted to right products of weight $n$ in the ideal $B$. In Section 5, we give the main results.

\section{Preliminaries}

Throughout this paper, $F$ will be a field of characteristic not 2. All vector spaces and algebras will be finite dimensional over $F$. Let $A$ be a nonassociative algebra and let us denote the bilinear product on $A$ by 
$A \times A \rightarrow A,(a, b) \mapsto a b$. Let $n \neq 0$ be a nonnegative integer, and let us denote by $\mathbb{I}(n)$ the set $\{1 ; 2 ; \cdots ; n\}$. If $A$ is a nonassociative algebra over $F$, the $F$-trilinear map $\{-,-,-\}: A \times A \times A \rightarrow A$ defined by $(x, y, z) \mapsto$ $(x y) z-x(y z)$ is called the associator of $A$.

If for all $(x, y, z)$ in $A^{3}$, we have $\{x, y, z\}=0$, the algebra $A$ is said associative.

Let $U, V, W$ be subspaces of $A .\{U, V, W\}$ is the subspace generated by the elements $\{u, v, w\}$, where $u \in U, v \in V$, and $w \in W$. Note $\operatorname{Assoc}(U)$ the following subspace:

$$
\operatorname{Assoc}(U)=\{U, A, A\}+\{A, U, A\}+\{A, A, U\} .
$$

A subspace $H$ of a nonassociative algebra $A$ is called left ideal (resp. right ideal) if for $x \in H$ and $a \in A$ one has $a x \in H$ (resp. $x a \in H$ ). An ideal is both left and right ideal. Let $\operatorname{Ide}(U)$ be the ideal of $A$ generated by the subalgebra $\operatorname{Assoc}(U)$.

For an ideal $B$ of a nonassociative algebra, we introduce the notations and following terminologies:

Let $P$ be a product of $m$ factors $s_{m}, s_{m-1}, \cdots, s_{1}$, that have been associated in an arbitrary way. We suppose that $n$ or more factors belong to $B$. We say that the product $P$ is of length $m$ and of weight $n$ with respect to the ideal $B$ or more simply that $P$ is of length $m$ and of weight $n$ in $B$. The length $m$ of $P$ will be noted $\#(P)$ and its weight $n$ will be noted $\#_{B}(P)$.

When $P=\left(\left(\cdots\left(\left(s_{m} s_{m-1}\right) s_{m-2} \cdots\right) s_{3}\right) s_{2}\right) s_{1}$ where the association is made always right, we say that $P$ is a right product and we write $P=s_{m} s_{m-1} s_{m-2} \cdots s_{1}$. Similarly, if $P=s_{1}\left(s_{2}\left(s_{3}\left(\cdots s_{m-2}\left(s_{m-1} s_{m}\right)\right) \cdots\right)\right)$ where the association is made always left, we say that $P$ is a left product. 
Let $S_{1}, S_{2}, \cdots, S_{p}$ be right products. One can write the right product (with $S_{j}$ as factors) $N=S_{p} S_{p-1} \cdots S_{1}$. We call $N$ a standard product.

Definition 2.1. Let $B$ be a subspace of the underlying vector space $A$.

- A subspace $B$ is right nilpotent if $B^{n}=\{0\}$ for some $n \geq 1$, where $B^{1}=B$ and $B^{n+1}=B^{n} \cdot B$. By convention, we set $B^{-n}=B^{0}=A$. Notice that $B^{n}$ is generated by right products of length $n$ and weight $n$ in $B$.

- A subspace $B$ is left nilpotent if ${ }^{n} B=0$ for some $n \geq 1$, where ${ }^{1} B=B$ and ${ }^{1+n} B=B \cdot\left({ }^{n} B\right)$. By convention, we set ${ }^{-n} B={ }^{0} B=A$. Notice that ${ }^{n} B$ is generated by left products of length $n$ and weight $n$ in $B$.

Definition 2.2. Let $B$ be an ideal of the nonassociative algebra $A$.

- Let $B^{\{n\}}$ be the subspace of the underlying vector space $A$ generated by all the products of length $n$ in $B$, associated in arbitrary way. We say that the ideal $B$ is nilpotent if there exists an integer $n$ such that $B^{\{n\}}=\{0\}$.

- Let $B^{\langle n\rangle}$ be the subspace generated by all products of elements in $A$ with at least $n$ elements in $B$. An ideal $B$ is strongly nilpotent if $B^{\langle n\rangle}=\{0\}$ for some $n \geq 1$.

Obviously, for all nonnegative integer $n, B^{\langle n\rangle}$ is an ideal of $A$ and one has $B \supseteq B^{\langle 1\rangle} \supseteq B^{\langle 2\rangle} \supseteq \cdots \supseteq B^{\langle n\rangle} \supseteq \cdots$ and $A^{n}$ (resp. ${ }^{n} A$ ) is a right ideal (resp. left ideal) of $A$.

We have also that $B^{\langle i\rangle} B^{\langle j\rangle} \subseteq B^{\langle i+j\rangle}$ for all nonnegative integers $i, j \geq 1$. 
Definition 2.3. Let $D$ be a subspace of the underlying vector space $A$ and $k$ be a nonnegative integer.

- $D_{(L, k)}$ is the vector subspace generated by all right products: " $d a_{k} \cdots a_{3} a_{2} a_{1}$ ", where $d$ belongs to $D$ and $a_{i}$ belongs to $A$ for any integer $i \in \mathbb{I}(k)$.

- ${ }_{(L, k)} D$ is the vector subspace generated by all left products: “ $a_{1}\left(a_{2}\left(a_{3}\left(\cdots\left(a_{k} d\right)\right)\right) \cdots\right)$ ", where $d$ belongs to $D$ and $a_{i}$ belongs to $A$ for any integer $i \in \mathbb{I}(k)$.

Definition 2.4. Let $B \neq\{0\}$ be an ideal of the nonassociative algebra $A$.

- We will say that $B$ is $A s s o_{k}$-right nil if there is an integer $k \geq 1$, such that the ideal $A \operatorname{ssoc}(B)$ satisfies $A \operatorname{ssoc}(B)_{(L, k)}=A \operatorname{ssoc}(B) \underbrace{L L L \cdots L}_{k \text {-times }}=\{0\}$.

- We will say that $B$ is $A s s o_{k}$-left nil if there is an integer $k \geq 1$, such that the ideal $A \operatorname{ssoc}(B)$ satisfies $(L, k) A \operatorname{ssoc}(B)=\underbrace{L \cdots L L L}_{k \text {-times }} A \operatorname{ssoc}(B)=\{0\}$.

Definition 2.5. Let $A$ be a nonassociative algebra and $B$ be an ideal in $A$. Let $a, b$ be two elements of $A$. If $a-b \in B$, we will say that $a \equiv b$ (modulo $B$ ).

\section{Right Products in the Algebra $A$}

Lemma 3.1. Let $A$ be a nonassociative algebra, $B$ be an ideal of $A$, and $U$ be subspace of $A$. For all $x$ in $U, b$ in $B$ and for all $a$ in $A$, $x b a-x(b a) \in\{U, B, A\} \subseteq A \operatorname{ssoc}(U)$.

Proof. Trivial, since $x b a-x(b a)=\{x, b, a\} \in \operatorname{Assoc}(U)$.

Lemma 3.2. For any integer $n \geq 3$,

$$
B^{n} \subseteq{ }^{n} B+A \operatorname{ssoc}(B) \text { and }{ }^{n} B \subseteq B^{n}+A \operatorname{ssoc}(B) .
$$


Proof.

$$
\begin{aligned}
& B^{n+1}=\left(B^{n-1} \cdot B\right) \cdot B \\
& \subseteq B^{n-1} \cdot(B \cdot B)+A \operatorname{ssoc}\left(B^{n-1}\right) \subseteq B^{n-1} \cdot(B \cdot B)+A \operatorname{ssoc}(B) \\
& \subseteq B^{n-1} \cdot\left({ }^{2} B\right)+A \operatorname{ssoc}(B) \subseteq\left(B^{n-2} B\right) \cdot\left({ }^{2} B\right)+A \operatorname{ssoc}(B) \\
& \subseteq B^{n-2} \cdot\left(B \cdot{ }^{2} B\right)+A \operatorname{ssoc}\left(B^{n-2}\right)+A \operatorname{ssoc}(B) \subseteq B^{n-2} \cdot\left({ }^{3} B\right)+A \operatorname{ssoc}(B) \\
& \subseteq\left(B^{n-3} B\right) \cdot\left({ }^{3} B\right)+A \operatorname{ssoc}(B) \\
& \quad \vdots \\
& \quad \cdots \\
& \subseteq\left(B^{3} \cdot B\right) \cdot\left({ }^{n-3} B\right)+A \operatorname{ssoc}(B) \\
& \subseteq B^{3} \cdot\left(B \cdot{ }^{n-3} B\right)+A \operatorname{ssoc}\left(B^{3}\right)+A \operatorname{ssoc}(B) \subseteq B^{3} \cdot\left({ }^{n-2} B\right)+A \operatorname{ssoc}(B) \\
& \subseteq\left(B^{2} \cdot B\right) \cdot\left({ }^{n-2} B\right)+A \operatorname{ssoc}(B) \\
& \subseteq B^{2} \cdot\left(B \cdot{ }^{n-2} B\right)+A \operatorname{ssoc}\left(B^{2}\right)+A \operatorname{ssoc}(B) \subseteq B^{2} \cdot\left({ }^{n-1} B\right)+A \operatorname{ssoc}(B) \\
& \subseteq(B \cdot B) \cdot\left({ }^{n-1} B\right)+A \operatorname{ssoc}(B) \\
& \subseteq B \cdot\left(B \cdot{ }^{n-1} B\right)+A \operatorname{ssoc}(B) \subseteq B \cdot\left({ }^{n} B\right)+A \operatorname{ssoc}(B) \\
& \subseteq{ }^{1+n} B+A s s o c(B) .
\end{aligned}
$$

Similarly, we can deduce that ${ }^{n} B \subseteq B^{n}+A \operatorname{ssoc}(B)$.

Let us notice that $B^{n}+\operatorname{Ide}(B) \subseteq{ }^{n} B+\operatorname{Ide}(B) \subseteq B^{n}+\operatorname{Ide}(B)$ since $\operatorname{Assoc}(B) \subseteq \operatorname{Ide}(B)$.

Lemma 3.3. Let $A$ be a nonassociative algebra and $B$ be an ideal of $A$. For all integer $k \geq 0, B^{k}$ and ${ }^{k} B$ satisfy:

$$
A \cdot B^{k} \subseteq B^{k}+\operatorname{Ide}(B) \supseteq B^{k} \cdot A \text { and } A \cdot{ }^{k} B \subseteq{ }^{k} B+\operatorname{Ide}(B) \supseteq{ }^{k} B \cdot A \text {. }
$$


Proof. For nonnegative integer $k$, we have

$$
\begin{aligned}
B^{k+1} \cdot A & \subseteq\left(B^{k} \cdot B\right) \cdot A \subseteq B^{k} \cdot(B \cdot A)+\left\{B^{k}, B, A\right\} \\
& \subseteq B^{k} \cdot B+\operatorname{Ide}(B)=B^{k+1}+\operatorname{Ide}(B)
\end{aligned}
$$

and

$$
\begin{aligned}
A \cdot{ }^{1+k} B & \subseteq A \cdot\left(B \cdot{ }^{k} B\right) \subseteq(A \cdot B) \cdot{ }^{k} B+\left\{A, B,{ }^{k} B\right\} \\
& \subseteq B \cdot{ }^{k} B+\operatorname{Ide}(B)={ }^{1+k} B+\operatorname{Ide}(B),
\end{aligned}
$$

also we have that

$$
\begin{aligned}
A \cdot B^{k+1} & \subseteq A \cdot\left({ }^{1+k} B+\operatorname{Ide}(B)\right) \subseteq A \cdot{ }^{1+k} B+\operatorname{Ide}(B) \\
& \subseteq{ }^{1+k} B+\operatorname{Ide}(B) \subseteq B^{k+1}+\operatorname{Ide}(B)
\end{aligned}
$$

and

$$
\begin{aligned}
{ }^{1+k} B \cdot A \subseteq & \left(B^{k+1}+\operatorname{Ide}(B)\right) \cdot A \subseteq B^{k+1} \cdot A+\operatorname{Ide}(B) \\
& \subseteq B^{k+1}+\operatorname{Ide}(B) \subseteq{ }^{1+k} B+\operatorname{Ide}(B) .
\end{aligned}
$$

Clearly, we have $B^{k+1} \subseteq B^{k},{ }^{1+k} B \subseteq{ }^{k} B$ and from Lemma 3.3, we can deduce the following lemma:

Lemma 3.4. Let $A$ be a nonassociative algebra and $B$ be an ideal of $A$. Let us define $B_{0}=A, B_{1}=B$ and $B_{k}=B^{k}+\operatorname{Ide}(B)={ }^{k} B+\operatorname{Ide}(B)$ for all integer $k \geq 2 ; B_{k}$ is an ideal of $A$, which satisfies $B_{k} \supseteq B_{k+1}$.

Proof. Naturally, we have

$$
\begin{aligned}
& A=B_{0} \supseteq B_{1} \supseteq B_{2} \supseteq \cdots \supseteq B_{k} \supseteq B_{k+1} \cdots . \\
& A={ }_{0} B \supseteq{ }_{1} B \supseteq{ }_{2} B \supseteq \cdots \supseteq{ }_{k} B \supseteq{ }_{1+k} B \cdots .
\end{aligned}
$$


Proposition 3.5. For given right product $P_{0}=a_{m} a_{m-1} a_{m-2} \cdots a_{3} a_{2} a_{1}$ and $Q_{0}$ an arbitrary product of length $\mathrm{m}^{\prime}$.

Let us set for any integer $i \in \mathbb{I}(m-1)$,

$$
P_{i}=a_{m} a_{m-1} \cdots a_{i+1}=\prod_{j=1}^{m-i} a_{m-j+1} .
$$

Then

$$
\begin{aligned}
T_{m}= & Q_{0} P_{0}=Q_{0} a_{m} a_{m-1} \cdots a_{2} a_{1} \\
& -\sum_{k=2}^{m-1}\left\{Q_{0}, P_{m-k+1}, a_{m-k+1}\right\} a_{m-k} \cdots a_{1}+\left\{Q_{0}, P_{1}, a_{1}\right\} .
\end{aligned}
$$

Proof. First of all let us define the following products: Let $m$ be the length of the product $P$.

For $i \in \mathbb{I}(m-1)$, let us define $a_{m-i+1}^{\prime}=a_{m-i+2}$. So we can write

$$
P_{i}^{\prime}=\prod_{j=1}^{m-i} a_{m-j+1}^{\prime}=\prod_{j=1}^{m-i} a_{m-j+2}=\prod_{j=1}^{m+1-(i+1)} a_{m+1-j+1}=P_{i+1}
$$

Now we can compute the proof: If $m=2$, one has

$$
\begin{aligned}
T_{2} & =Q_{0}\left(a_{2} a_{1}\right)=Q_{0} a_{2} a_{1}-\left\{Q_{0}, a_{2}, a_{1}\right\}=Q_{0} a_{2} a_{1}-\left\{Q_{0}, P_{1}, a_{1}\right\}, \text { and if } m=3 ; \\
T_{3} & =Q_{0}\left(a_{3} a_{2} a_{1}\right) \\
& =Q_{0}\left(a_{3} a_{2}\right) a_{1}-\left\{Q_{0}, a_{3} a_{2}, a_{1}\right\}=\left(Q_{0}\left(a_{3} a_{2}\right)\right) a_{1}-\left\{Q_{0}, P_{1}, a_{1}\right\} \\
& =\left(Q_{0} a_{3} a_{2}-\left\{Q_{0}, a_{3}, a_{2}\right\}\right) a_{1}-\left\{Q_{0}, P_{1}, a_{1}\right\} \\
& =Q_{0} a_{3} a_{2} a_{1}-\left\{Q_{0}, P_{2}, a_{2}\right\} a_{1}-\left\{Q_{0}, P_{1}, a_{1}\right\} .
\end{aligned}
$$

With the assumption that for a right product $P_{0}$ of length $m$, one has

$$
\begin{aligned}
T_{m}=Q_{0} P_{0} & =Q_{0} a_{m} a_{m-1} \cdots a_{2} a_{1} \\
& -\sum_{k=2}^{m-1}\left\{Q_{0}, P_{m-k+1}, a_{m-k+1}\right\} a_{m-k} \cdots a_{1}+\left\{Q_{0}, P_{1}, a_{1}\right\}
\end{aligned}
$$


Then let us consider a right product $P$ of length $m+1$, say

$$
\begin{aligned}
P=a_{m+1} a_{m} a_{m-1} \cdots a_{3} a_{2} a_{1}: & \\
T_{m+1} & =Q_{0} P=Q_{0} \prod_{k=1}^{m+1} a_{m-k+2}=Q_{0}\left(\prod_{k=1}^{m} a_{m-k+2} a_{1}\right) \\
& =Q_{0} \prod_{k=1}^{m} a_{m-k+2} a_{1}-\left\{Q_{0}, \prod_{k=1}^{m} a_{m-k+2}, a_{1}\right\} \\
& =Q_{0} P_{0}^{\prime} a_{1}-\left\{Q_{0}, P_{0}^{\prime}, a_{1}\right\} .
\end{aligned}
$$

By the assumption, $Q_{0} P_{0}^{\prime}$ (since the length of $P_{0}^{\prime}$ is $m$ ) can be written as:

$$
\begin{aligned}
T_{m}^{\prime}= & Q_{0} P_{0}^{\prime}=Q_{0} a_{m}^{\prime} a_{m-1}^{\prime} \cdots a_{2}^{\prime} a_{1}^{\prime} \\
& -\sum_{k=2}^{m-1}\left\{Q_{0}, P_{m-k+1}^{\prime}, a_{m-k+1}^{\prime}\right\} a_{m-k}^{\prime} \cdots a_{1}^{\prime}-\left\{Q_{0}, P_{1}^{\prime}, a_{1}^{\prime}\right\} .
\end{aligned}
$$

Thanks to Equation (3.2), we have

$$
\begin{aligned}
& T_{m+1}=Q_{0} P_{0}^{\prime} a_{1}-\left\{Q_{0}, P_{0}^{\prime}, a_{1}\right\} \\
& =\left(Q_{0} a_{m}^{\prime} a_{m-1}^{\prime} \cdots a_{2}^{\prime} a_{1}^{\prime}-\sum_{k=2}^{m-1}\left\{Q_{0}, P_{m-k+1}^{\prime}, a_{m-k+1}^{\prime}\right\} a_{m-k}^{\prime} \cdots a_{1}^{\prime}-\left\{Q_{0}, P_{1}^{\prime}, a_{1}^{\prime}\right\}\right) \\
& \times a_{1}-\left\{Q_{0}, P 1_{0}, a_{1}\right\} \\
& =\left(Q_{0} a_{m+1} a_{m} \cdots a_{3} a_{2}-\sum_{k=2}^{m-1}\left\{Q_{0}, P_{m-k+2}, a_{m-k+2}\right\} a_{m-k+1} \cdots a_{2}-\left\{Q_{0}, P_{2}, a_{2}\right\}\right) \\
& \times a_{1}-\left\{Q_{0}, P_{1}, a_{1}\right\} .
\end{aligned}
$$


It follows that

$$
\begin{aligned}
T_{m+1}= & Q_{0} a_{m+1} a_{m} \cdots a_{3} a_{2} a_{1}-\left\{Q_{0}, P_{1}, a_{1}\right\} \\
& \quad-\sum_{k=2}^{m-1}\left\{Q_{0}, P_{m-k+2}, a_{m-k+2}\right\} a_{m-k+1} \cdots a_{2} a_{1}-\left\{Q_{0}, P_{2}, a_{2}\right\} a_{1} \\
= & Q_{0} a_{m+1} a_{m} \cdots a_{3} a_{2} a_{1}-\left\{Q_{0}, P_{1}, a_{1}\right\} \\
& \quad-\sum_{k=2}^{m+1-2}\left\{Q_{0}, P_{m+1-k+1}, a_{m+1-k+1}\right\} a_{m+1-k} \cdots a_{2} a_{1} \\
= & Q_{0} a_{m+1} a_{m} \cdots a_{3} a_{2} a_{1}-\left\{Q_{0}, P_{1}, a_{1}\right\} \\
& \quad-\sum_{k=2}^{m-1}\left\{Q_{0}, P_{m+1-k+1}, a_{m+1-k+1}\right\} a_{m+1-k} \cdots a_{2} a_{1} .
\end{aligned}
$$

The proof is done.

Lemma 3.6. Any product $T$ with length $m$ in a nonassociative algebra $A$ is a linear combination of right products of length $m$ modulo the ideal $\operatorname{Ide}(A)$.

Proof. By induction on the length $m$, we have: If $m$ equals 1 or 2 , there is nothing to do. If $m=3$, one can notice that $T=a b c$ or $a(b c)=$ $a b c-\{a, b, c\}$ for all $a, b, c$ in $A$. The lemma is also obvious.

Let us suppose that the lemma is true for a product which length is strictly less than $m^{\prime} \geq 4$.

Now for a given product (with length $m^{\prime}$ ) $T=Q_{0} P_{0}$, where $P_{0}$ is a right product of length $m$ such that $m^{\prime}>m \geq 1$. Thanks to Proposition 3.5,

$$
\begin{aligned}
T= & Q_{0} P_{0}=Q_{0} a_{m} a_{m-1} \cdots a_{2} a_{1} \\
& -\sum_{k=2}^{m-1}\left\{Q_{0}, P_{m-k+1}, a_{m-k+1}\right\} a_{m-k} \cdots a_{1}-\left\{Q_{0}, P_{1}, a_{1}\right\}
\end{aligned}
$$




$$
\begin{gathered}
=Q_{0} a_{m} a_{m-1} \cdots a_{2} a_{1}-\sum_{k=2}^{m-1} Q_{0} P_{m-k+1} a_{m-k+1} a_{m-k} \cdots a_{1} \\
+\sum_{k=2}^{m-1} Q_{0}\left(P_{m-k+1} a_{m-k+1}\right) a_{m-k} \cdots a_{1}-\left\{Q_{0}, P_{1}, a_{1}\right\} .
\end{gathered}
$$

Let $k \in \mathbb{I}(m-2)$. The length of the products $Q_{0} P_{m-k} a_{m-k}$, $Q_{0}\left(P_{m-k} a_{m-k}\right), Q_{0}$ are less or equal to $m-1$, so, they are linear combinations of right products modulo the ideal $\operatorname{Ide}(A)$. This let us know that $T=Q_{0} P_{0}$ is a linear combination of right products of length $m$ modulo the ideal $\operatorname{Ide}(A)$.

\section{Right Products of Weight $n$ in the Ideal $B$}

Lemma 4.1. Any product $T$ with length $m$ and weight $n \geq 1$ with regard to the ideal $B$ of nonassociative algebra $A$ is a linear combination of right products of length $m$ and weight $n$ modulo the ideal Ide(B).

Proof. By induction on the length $m$, we have

The lemma is obvious if $m \leq 2$. If $m=3$, then there are $a_{1}, a_{2}, a_{3}$ elements in $A$ such that $P$ is one of the following: $a_{3} a_{2} a_{1}$ and $a_{3}\left(a_{2} a_{1}\right)=$ $a_{3} a_{2} a_{1}-\left\{a_{3}, a_{2}, a_{1}\right\}$. So for $m=3, P$ is a linear combination of right products modulo the ideal $\operatorname{Ide}(B)$.

Let us suppose that the lemma is true for a product which length is strictly less than $m^{\prime} \geq 4$.

Now for a given product (with length $m^{\prime}$ and weight $n \geq 1$ ) $T=Q_{0} P_{0}$, where $P_{0}$ is a right product of length $m$ such that $m^{\prime}>m$. Thanks to the Proposition 3.5,

$$
\begin{aligned}
T= & Q_{0} P_{0}=Q_{0} a_{m} a_{m-1} \cdots a_{2} a_{1} \\
& -\sum_{k=2}^{m-1}\left\{Q_{0}, P_{m-k+1}, a_{m-k+1}\right\} a_{m-k} \cdots a_{1}-\left\{Q_{0}, P_{1}, a_{1}\right\}
\end{aligned}
$$




$$
\begin{aligned}
= & Q_{0} a_{m} a_{m-1} \cdots a_{2} a_{1}-\sum_{k=2}^{m-1} Q_{0} P_{m-k+1} a_{m-k+1} a_{m-k} \cdots a_{1} \\
& +\sum_{k=2}^{m-1} Q_{0}\left(P_{m-k+1} a_{m-k+1}\right) a_{m-k} \cdots a_{1}-\left\{Q_{0}, P_{1}, a_{1}\right\} .
\end{aligned}
$$

Let $k \in \mathbb{I}(m-2)$, we know that $Q_{0} P_{m-k} a_{m-k}, Q_{0}\left(P_{m-k} a_{m-k}\right)$ and $Q_{0}$ are products, which length are less or equal to $m^{\prime}-1$. So by induction, they are linear combinations of right products modulo the ideal $\operatorname{Ide}(B)$.

This let us know that $T=Q_{0} P_{0}$ is a linear combination of right products modulo the ideal $\operatorname{Ide}(B)$.

Through the calculation, it is clear that the terms (for $k$ in $\mathbb{I}(m-2))$ : $Q_{0} P_{m-k} a_{m-k} a_{m-k-1} \cdots a_{1}, Q_{0}\left(P_{m-k} a_{m-k}\right) a_{m-k-1} \cdots a_{1}$ and $Q_{0} a_{m} a_{m-1} \cdots a_{2} a_{1}$ keep invariant the length $m^{\prime}$ and weight $n$ of $T=Q_{0} P_{0}$.

Lemma 4.2. Let $A$ be a nonassociative algebra and $B \neq\{0\}$ be an ideal in $A$. Let $P_{0}=a_{m} a_{m-1} a_{m-2} \cdots a_{3} a_{2} a_{1}$ be a right product with length $m$ and weight $n \geq 1$ in $B$. Then $P_{0}$ belongs to $B_{n}$.

Proof. Let $\sigma$ be an injective map of $\mathbb{I}(n)$ to dans $\mathbb{I}(m)$ such that $i<j$ implies that $\sigma(i)<\sigma(j)$ and for all $j \in \mathbb{I}(n), a_{\sigma(j)} \in B$. Let us also define for all integer $k \in \mathbb{I}(n-1)$, the following products:

$$
\begin{aligned}
& Q_{0}^{\prime}=a_{m} a_{m-1} \cdots a_{\sigma(n)+1}, \\
& Q_{k}=Q_{k-1}^{\prime} a_{\sigma(n-k+1)}, \\
& Q_{k}^{\prime}=Q_{k} a_{\sigma(n-k+1)-1} \cdots a_{\sigma(n-k)+1}, \\
& Q_{n}=Q_{n-1}^{\prime} a_{\sigma(1)} \cdots a_{1} .
\end{aligned}
$$

Clearly, $Q_{1}$ belongs to $B \subseteq B^{1}+\operatorname{Ide}(\{B, A, A\})=B_{1}$ and also $Q_{1}^{\prime}$ belongs to $B_{1}$. 
Since $B_{1}$ is an ideal $Q_{2}=Q_{1}^{\prime} a_{\sigma(n-j+1)}$ belongs to $B \cdot B \subseteq B^{2}+\operatorname{Ide}(B)=B_{2}$. By induction, let us suppose that for integer $j$ such that $1 \leq j<n$, we have $Q_{j}$ belongs to $B_{j}=B^{j}+\operatorname{Ide}(B)$. Then let us show that $Q_{j+1}$ is an element of $B_{j+1}=B^{j+1}+\operatorname{Ide}(B)$.

Indeed, we have $Q_{j}^{\prime}=Q_{j} a_{\sigma(n-j+2)-1} \cdots a_{\sigma(n-j+1)+1}$ is an element of the ideal $B_{j}$ and so on, $Q_{j+1}=Q_{j}^{\prime} a_{\sigma(n-j+1)}$ belongs to $B_{j} \cdot B \subseteq B^{j+1}+$ $\operatorname{Ide}(B)=B_{j+1}$. Then we have proved that $P_{0}=Q_{n}$ is an element of $B_{n}$.

Lemma 4.3. Let $l, k$ be integers such that $1 \leq k \leq l$ and let $A$ be a nonassociative algebra and $B$ be an ideal of $A$ which is Es $s_{k}$-right nil. $A$ right product $P=a_{m} a_{m-1} a_{m-2} \cdots a_{3} a_{2} a_{1}$, of length $m$ and weight (in B) $n$ greater or equal to $2 l$, belongs to $B_{(L, k)}^{l}$.

Proof. The right product $Q=a_{m} a_{m-1} a_{m-2} \cdots a_{k+1}$ is of weight greater or equals to $l$, indeed let $n^{\prime}$ be the weight of $Q$ and $n^{\prime \prime}$ be the weight of $a_{k} \cdots a_{3} a_{2} a_{1}$. We have $0 \leq n^{\prime \prime} \leq k$ and the equality $P=Q a_{k} \cdots$ $a_{3} a_{2} a_{1}$ implies that $n^{\prime} \leq n \leq k+n^{\prime}$. So $n^{\prime} \geq n-k \geq 2 l-k \geq l$. The Lemma 4.2 tells that $Q \in B_{l}$. And so on, $P=Q a_{k} \cdots a_{3} a_{2} a_{1} \in\left(B_{l}\right)_{(L, k)}=$ $\left(B^{l}+\operatorname{Ide}(\{B, A, A\})\right)_{(L, k)}=B_{(L, k)}^{l}$ since $B$ is an ideal $A \operatorname{ssoc}_{k}$-right nil. $\square$

Lemma 4.4. Let $k$ be an integer such that the ideal $B$ is $E s_{k}$-right nil. Let $P$ be product of weight $t \geq 4 k^{2}-2 k+1$ with regard to the ideal $B$, then $P$ is a linear combination of right products $Q_{j}$ such that, for any $j$, we have $Q_{j}$ belongs to $B_{(L, k)}^{k}$ or has at least one factor in $B_{(L, k)}^{k}$. 
Proof. Let $k>1$ and $t \geq 4 k^{2}-2 k+1$. Thank's to the Lemma 4.1, any product $P$ of weight greater or equals to $t$ is a linear combination of right products of weight greater or equals to $t$. Let $P=\sum_{j} \mu_{j} Q_{j}$, where $Q_{j}$ is a right product of weight greater or equals to $t$.

For any $j$, we have $Q_{j}=s_{j, p} s_{j, p-1} \cdots s_{j, 1}$, where $s_{j, i} \in L$ ( $p$ is the length of $Q_{j}$ ).

- If, there is one element $s_{j, i_{0}}$ such that it's weight is greater or equals to $2 k$, then $s_{j, i_{0}}$ belongs to $B_{(L, k)}^{k}$ by application of the Lemma 4.3. And so on $Q_{j}$ has a factor in $B_{(L, k)}^{k}$.

- Else, every factor $s_{j, i}$ has a weight strictly less than $2 k$. Let $q$ be the number of factors $s_{j, i}$ with a weight greater or equals to 1 . Then one has $q(2 k-1) \geq t=4 k^{2}-2 k+1$ and then $q>2 k$.

When the weight of $s_{j, i}$ is greater or equals to 1 , we have $s_{j ; i}$ belongs to $B$. So $Q_{j}$ is of weight greater or equals to $q$. Since $q \geq 2 k$, we have $Q_{j} \in B_{(L, k)}^{k}$ thank's to Lemma 4.3.

By abuse, we will say that $Q_{j}$ has at least one factor in $B_{(L, k)}^{k}$.

\section{Main Theorem}

Theorem 5.1. Let $F$ be a commutative field with characteristic not 2, $A$ be an F-nonassociative algebra, and $B$ be an ideal of $A$ which is $E s_{k}$-right-nil. Then the following assertions are equivalent:

(i) $B$ is right nilpotent;

(ii) B is nilpotent;

(iii) B is strongly nilpotent. 
Proof. Indeed, for any integer $k \geq 1$, the vectors spaces's inclusions $B^{k} \subseteq B^{\{k\}} \subseteq B^{\langle k\rangle}$ tell us that (iii) $\Rightarrow$ (ii) $\Rightarrow$ (i).

Furthermore, suppose that there is an integer $l^{\prime} \geq 1$ such that $B^{l^{\prime}}=\{0\}$. Let us define $k=\max \left\{k^{\prime}, l^{\prime}\right\}$, then for an integer $A$ such that $l=4 k^{2}-2 k+1$, the Lemma 4.4 tells us that any product $P$ with weight greater or equals to $l=4 k^{2}-2 k+1$, in $B$ is a linear combination of right products which have at least one factor in $\left(B^{k}\right)_{(L, k)} \subseteq\left(B^{k^{\prime}}\right)_{(L, k)}=\{0\}$. And so on $P=0$. Then $B^{\langle l\rangle}=0$. The implication (i) $\Rightarrow$ (iii) is done.

Corollary 5.2. Let $F$ be a commutative field with characteristic not 2 and $A$ be an nonassociative F-algebra. The following assertions are equivalent:

(i) $A$ is right nilpotent;

(ii) A is nilpotent;

(iii) $A$ is strongly nilpotent.

Proof. Clearly, we have (iii) $\Rightarrow$ (ii) $\Rightarrow$ (i).

Let us notice that $A \operatorname{ssoc}(L)$ is a subset of $A^{3}$ and if there is an integer $l^{\prime} \geq 1$ such that $A^{l^{\prime}}=\{0\}$, we do have that $\operatorname{Assoc}(L)$ is an ideal Asso $l^{\prime}$-right-nil. The Theorem 5.1 tells us that (i) $\Rightarrow$ (iii).

Corollary 5.3. Let $F$ be a commutative field with characteristic not 2 and $A$ be an nonassociative $F$-algebra. The following assertions are equivalent:

(i) A is left nilpotent;

(ii) A is nilpotent;

(iii) A is strongly nilpotent. 
Proof. From the algebra $A$, with bilinear product on $A$ by $\phi: A \times A \rightarrow A$, $(a, b) \mapsto \phi(a, b)=a b$ we can obtain the algebra $A^{o p}$ where bilinear product of $a$ and $b$ is $\phi(b, a)$. Then, left nilpotency of $A$ is equivalent of right nilpotency of $A^{o p}$.

Remark 5.4. Right nilpotency of an ideal $B$ of $A$ may not implies left nilpotency of the ideal $B$ and vice-versa. For the (left) Leibniz algebra $A$ defined in Example 3.3 of [3], the ideal $I=E s s(L)$ generated by all squares is $E s_{1}$-right-nil.

But for all integer $k,(A, k) A \operatorname{ssoc}(I)=\underbrace{A \cdots A A A}_{k \text {-times }} A \operatorname{ssoc}(I) \neq\{0\}$.

\section{References}

[1] C. J. A. Béré, N. B. Pilabré and M. Ouattara, Nilpotence dans les algebres de Malcev, soumis à publication.

[2] C. J. A. Béré, M. F. Ouedraogo and M. Ouattara, On nilpotency in Leibniz algebras, soumis à publication.

[3] C. J. A. Béré, A. Kobmbaye and A. Konkobo, On a class of Leibniz algebras, International Journal of Advanced Mathematical Sciences 3(2) (2015), 147-155.

www.sciencepubco.com/index.php/IJAMS

(C) Science Publishing Corporation doi:10.14419/ijams.v3i2.5290

[4] C. J. A. Béré, N. B. Pilabré and A. Kobmbaye, Lie's theorems on soluble Leibniz algebras, British Journal of Mathematics \& Computer Science 4(18) (2014), 2570-2581.

[5] K. A. Zhevlakov, A. M. Slin'ko, I. P. Shestakov and A. I. Shirshov, Rings that are Nearly Associative, Translated by Harry F. Smith, Academic Press, New York, 1982, xi +371 pp., ISBN $0-1277-9850-1$. 